Research Article

\title{
Feasibility of Implementing Therapeutic Yoga in Assisted Living Facilities
}

Em V Adams ${ }^{1,}{ }^{*}$, Marieke Van Puymbroeck ${ }^{2}$, Brandi M Crowe ${ }^{3}$, Cynthia LS Pury ${ }^{4}$, Claire K Allison ${ }^{5}$, Arlene A Schmid ${ }^{6}$

1. Herbert H. Lehman College, Bronx, New York, 10468, USA; E-Mail: emilie.adams@lehman.cuny.edu

2. Clemson University, 282b Lehotsky, Clemson, SC, 29634 USA; E-Mail: mvp@clemson.edu

3. Clemson University, 278 Lehotsky Hall, Clemson, SC 29634 USA; E-Mail: bmcrowe@clemson.edu

4. Clemson University, 410 H Brackett Hall, Clemson, SC 29634 USA; E-Mail: CPURY@clemson.edu

5. Clemson University, Clemson, SC 29634 USA; E-Mail: ckelly6@g.clemson.edu

6. Colorado State University, Fort Collins, CO USA; E-Mail: Arlene.schmid@colostate.edu

* Correspondence: Em V Adams; E-Mails: emilie.adams@lehman.cuny.edu; emiliea@clemson.edu

Academic Editor: Marieke Van Puymbroeck

Special Issue: Yoga in Older Adults

OBM Geriatrics

2021, volume 5, issue 1

doi:10.21926/obm.geriatr.2101148
Received: August 19, 2020

Accepted: December 22, 2020

Published: January 04, 2021

\begin{abstract}
The purpose of this convergent mixed methods study was to explore the feasibility of implementing a chair yoga intervention in assisted living facilities (ALFs), evaluating reach, demand, acceptability, practicality, treatment fidelity, and safety. A yoga intervention was implemented twice a week for eight weeks at four different ALFs. Feasibility data were collected in the form of observational data, reflective field notes, and focus groups. Quantitative and qualitative data strands were compared to determine if results were convergent or divergent. Quantitative benchmarks were met for reach, demand, acceptability, practicality, treatment fidelity, and safety. A directed content analysis revealed qualitative results were convergent with quantitative results. Yoga appears to be feasible, but practicality varied widely and was largely dependent on the resources (i.e., staff support and space)
\end{abstract}

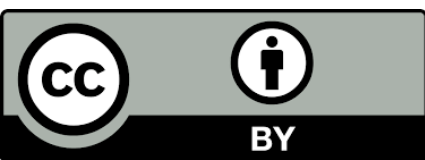

(C) 2021 by the author. This is an open access article distributed under the conditions of the Creative Commons by Attribution License, which permits unrestricted use, distribution, and reproduction in any medium or format, provided the original work is correctly cited. 
available at individual facilities. Recommendations for future research and practical implications are discussed.

\section{Keywords}

Yoga; feasibility; long-term care; older adults; recreational therapy

\section{Introduction}

The number of older adults in the United States is increasing, and correspondingly, so is the number of people receiving long-term care, including assisted living facility (ALF) services [1, 2]. Older adults who have personal care or health care needs that do not warrant the level of care offered by skilled nursing facilities, may move to an ALF. ALFs provide meals, medication management, assistance with activities of daily living, structured social and recreation programs, and 24-hour staff support as needed [1]. The purpose of ALFs it to promote autonomy and quality of life [3], but generally speaking, residents of ALFs have higher rates of pain [4,5], lower levels of functional fitness $[6,7]$, and lower perceived social support than their community-dwelling peers [8]. Recreational therapists and activity directors in ALF settings are tasked with implementing social and recreation programs that are both of interest to residents, and that will help improve or maintain physical, cognitive, and psychosocial functioning [9].

While many interventions are used that focus on single domains of functioning (i.e. silver sneakers to improve/maintain physical functioning, social groups to reduce physical isolation), yoga is uniquely situated to simultaneously improve multiple health domains [10]. Yoga is an eastern practice that incorporates asana (postures), pranayama (breathwork), and dhyana (meditation) [10]. There is a large body of research demonstrating the effectiveness of yoga as an integrative therapy to improve pain management [11, 12], improve balance [12-15], increase functional fitness [16, 17], and increase social support $[14,18]$. Additionally, yoga continues to grow in popularity among the general population, indicating there may be a growing interest among residents of ALFs as well [19]. Among community-dwelling older adults, researchers have found that yoga interventions have yielded these benefits for individuals with a variety of chronic illnesses, including chronic stroke [2022], Parkinson's disease [23-25], multiple sclerosis [26, 27], traumatic brain injury [28, 29], and diabetic peripheral neuropathy $[12,14]$.

However, the current literature on the effectiveness of yoga has either been conducted with healthy community-dwelling adults, or tailored to specific health conditions. Residents of ALFs who have a wide range of health conditions and abilities may require various modifications of the yoga practice to make it accessible for all residents of ALFs. While yoga postures can be adapted to be inclusive of all levels of cognitive and physical functioning without changing the core nature of the activity; the feasibility of implementing this type of adapted practice in an ALF is unknown [30].

To our knowledge, there has only been one study of yoga in an ALF. This study examined the impact of yoga on the physical fitness (cardiovascular function, flexibility, strength, and balance), sleep quality, and depression levels of transitional frail elders residing in an ALF in Taiwan [31, 32]. However, there were serious methodological concerns with the study, particularly regarding the qualifications of the instructor/s who were not adequately trained or qualified to instruct yoga. The 
minimum hours required for credentialing by Yoga Alliance is 200 hours [33]; while the instructors for the Taiwanese study only received nine hours of training [34]. The instructor used pre-recorded verbal instructions for the class and the instructor's primary responsibility was to take notes of any "signs or symptoms of discomfort" (p. 55) that occurred during the sessions. The manuscript did not specify whether modifications were provided or if steps were taken to address and reduce signs of discomfort. Using inadequately trained instructors compromises potential benefits and places participants at an increased risk of injury [35]. Thus, the results from this study did not adequately demonstrate the feasibility of yoga in ALFs.

Feasibility studies examine specific components of an intervention without controlled settings to determine if the intervention remains effective when implemented in less than ideal circumstances [36]. Feasibility studies are warranted if evidence-based interventions are conducted in different settings, or if the target population has unique considerations [36]. Testing feasibility in the specified environment is important to ensure the intervention could be successfully conducted as planned. Aspects of feasibility include reach, demand, acceptability, practicality, treatment fidelity, and safety (see Table 1 for the full definition of each feasibility construct) [36]. Because ALFs serve people with diverse health conditions, there is a need to determine if it is feasible to implement a generalized therapeutic yoga intervention for residents of ALFs that will be safe and provide benefits for all participants. Therefore, the purpose of this study was to evaluate the feasibility of implementing a twice-weekly therapeutic yoga intervention for eight weeks in ALFs.

Table 1 Descriptions of feasibility components and qualitative codes.

\begin{tabular}{|c|c|c|}
\hline $\begin{array}{l}\text { Feasibility } \\
\text { Component }\end{array}$ & Description & Pre-determined qualitative codes \\
\hline Reach & $\begin{array}{l}\text { Portion of the target population that } \\
\text { engages in the intervention [36] }\end{array}$ & $\begin{array}{l}\text { Initial expectations, initial } \\
\text { concerns, and motivations to } \\
\text { participate }\end{array}$ \\
\hline Demand & $\begin{array}{l}\text { The extent to which the intervention is } \\
\text { likely to be used [36] }\end{array}$ & $\begin{array}{l}\text { Intent to continue yoga practice } \\
\text { and current independent yoga } \\
\text { practice }\end{array}$ \\
\hline Acceptability & $\begin{array}{l}\text { How appealing and appropriate the } \\
\text { population perceived the intervention } \\
{[36]}\end{array}$ & $\begin{array}{l}\text { Positive and negative } \\
\text { programmatic components and } \\
\text { perceived outcomes }\end{array}$ \\
\hline Treatment fidelity & $\begin{array}{l}\text { The quantity and quality of components } \\
\text { in the delivered intervention compared } \\
\text { to the planned intervention [36] }\end{array}$ & $\begin{array}{l}\text { Justification of modifications to } \\
\text { the protocol }\end{array}$ \\
\hline Practicality & $\begin{array}{l}\text { Factors that contribute to the ease or } \\
\text { difficulty of implementation [36] }\end{array}$ & $\begin{array}{l}\text { Facilitators for and barriers to } \\
\text { implementation }\end{array}$ \\
\hline Safety & $\begin{array}{l}\text { Adverse events, or potential adverse } \\
\text { events that occur during implementation } \\
\text { [36] }\end{array}$ & Perceived safety \\
\hline
\end{tabular}




\section{Methods}

\subsection{Design of the Study}

The data from this study were part of a larger study, approved by a university institutional review board (approval number IRB2019-005) that employed a single-arm convergent mixed methods research design to evaluate the feasibility and impact of an eight-week yoga intervention in multiple ALFs. The present analysis focused solely on the feasibility aspect of the larger study.

\subsection{Facilities}

The principal investigator (PI) recruited participants from four ALFs in the Southeast region of the United States. Two of the facilities (Facility A and B) were solely ALFs, and two (Facility C and D) were part of larger LTC complexes that also offered memory care and independent living services. Regular programming at each of the facilities included at least three sessions of physical activity a week, but yoga and other mindfulness-based physical activity had not been incorporated within the past five years. In facility $A$, the only space large enough to accommodate the intervention was an activity room, an open space off the main hallway. In facilities $B$ and $C$, the intervention was implemented in the dining hall; one was an enclosed room, and the other an open space between two wings of the building. Finally, in Facility $D$, the intervention was held in the chapel, a small enclosed space and secluded room on the main floor.

\subsection{Recruitment}

The target population for the yoga intervention was current residents of the ALFs. Recruitment took place at each ALF with the assistance of the respective Activity Directors. The PI met with potential participants, explained the intervention, and obtained informed consent from interested individuals. To screen for eligibility, the PI used the eight-item Physical Activity Readiness Questionnaire (PAR-Q) to determine if potentially eligible individuals had any contraindications for physical activity [37]. Individuals who endorsed one or more items needed physician approval prior to participation in the intervention. The MiniCOG, a four-item cognitive assessment that involves drawing a clock and completing a three-word recall, was used to screen for cognitive impairment. Possible scores range from 0 (no questions correct) to 5 (all questions correct), and individuals who scored below three (indicating the need for additional cognitive impairment screening) were excluded from the study [38]. Additionally, to be included in the study potential participants must have committed to attending yoga twice a week for eight weeks. Potential participants were excluded if they were expecting to leave the facility within 10 weeks, had any known upcoming surgeries that would occur during the intervention, were unwilling to provide informed consent, had a known diagnosis of dementia, or required ventilator assistance to breathe. See Table 2 for more specific details regarding differences in recruitment between facilities.

Table 2 Recruitment diagram and facility information.

\begin{tabular}{llllll}
\hline & Facility A & Facility B & Facility C & Facility D & Total \\
\hline Levels of Care Offered & $\mathrm{AL}$ & $\mathrm{AL}$ & $\mathrm{IL}, \mathrm{AL}, \mathrm{MC}$ & $\mathrm{AL}, \mathrm{MC}$ & \\
\hline
\end{tabular}




\begin{tabular}{|c|c|c|c|c|c|}
\hline $\begin{array}{l}\text { Number of Residents } \\
\text { (Female, Male) }\end{array}$ & $\begin{array}{l}42 \\
(34,8)\end{array}$ & $\begin{array}{l}34 \\
(30,4)\end{array}$ & $\begin{array}{l}33 \\
(24,9)\end{array}$ & $\begin{array}{l}17 \\
(11,6)\end{array}$ & $\begin{array}{l}126 \\
(99,27)\end{array}$ \\
\hline $\begin{array}{l}\text { Did not contact due to } \\
\text { known dementia diagnosis }\end{array}$ & 0 & 17 & 0 & 0 & 17 \\
\hline $\begin{array}{l}\text { Contacted to assess for } \\
\text { eligibility }\end{array}$ & 42 & 17 & 33 & 17 & 109 \\
\hline $\begin{array}{l}\text { Unwilling to provide } \\
\text { informed consent }\end{array}$ & 32 & 13 & 25 & 10 & 80 \\
\hline $\begin{array}{l}\text { Number who signed } \\
\text { informed consent }\end{array}$ & 10 & 4 & 8 & 7 & 29 \\
\hline $\begin{array}{l}\text { Excluded due to upcoming } \\
\text { surgeries that will occur } \\
\text { during the study }\end{array}$ & 1 & 0 & 0 & 0 & 1 \\
\hline Scored below 3 on MiniCOG & 4 & 0 & 4 & 2 & 10 \\
\hline $\begin{array}{l}\text { Did not commit to attend } \\
\text { all yoga sessions }\end{array}$ & 0 & 0 & 0 & 1 & 1 \\
\hline Number enrolled & 5 & 4 & 4 & 4 & 17 \\
\hline Lost to follow-up & 1 & 0 & 1 & 0 & 2 \\
\hline Total Completed Study & 4 & 4 & 3 & 4 & 15 \\
\hline $\begin{array}{l}\text { Average number of } \\
\text { residents in attendance at } \\
\text { yoga }\end{array}$ & $8.88 \pm 2.19$ & $9.44 \pm 3.32$ & $4.33 \pm 2.13$ & $5.87 \pm 2.26$ & $7.19 \pm 3.26$ \\
\hline $\begin{array}{l}\text { Percentage of residents in } \\
\text { attendance }\end{array}$ & $21.14 \%$ & $27 \%$ & $13.12 \%$ & $34.52 \%$ & $23.95 \%$ \\
\hline $\begin{array}{l}\text { Was staff present during } \\
\text { intervention? }\end{array}$ & No & Yes & No & Yes & \\
\hline Location of intervention & $\begin{array}{l}\text { Activity } \\
\text { room }\end{array}$ & Dining hall & Dining hall & Chapel & \\
\hline
\end{tabular}

$A L=$ Assisted living, $\mathrm{IL}=$ Independent Living, $\mathrm{MC}=$ Memory Care Unit

\subsection{Intervention}

The protocol delineated an eight-week chair yoga intervention delivered by a Certified Yoga Therapist (C-IAYT) bi-weekly in 60-minute group sessions. Study participants, as well as residents who did not qualify for the study, attended the groups. Because the purpose of this study was to evaluate feasibility, it was important to include all residents who would typically engage in facility 
activities. Though not everyone met inclusion criteria to participate in the study, no residents were turned away from participating in yoga unless they had specific restrictions regarding physical activity. The intervention was developed by the PI based on previous sequences that were demonstrated to be acceptable for older adults with a variety of health conditions [24]. The sequence utilized traditional yoga postures that were modified for a seated practice. Each session adhered to the following general sequence: 1) grounding movement; 2) mantra (dhyana); 3) breath practice (pranayama); 4) warming sequence (asana); 5) postures (asana) including movement across the midline, strength, flexibility and balance postures; 6) moving meditation (dharana); 7) moment of stillness (pratyahara); and 8) a moment of connection (dyhana). A research assistant, who was a Certified Therapeutic Recreation Specialist, observed each session either in person or via video recording to complete the treatment fidelity checklist. Chairs were arranged in a circle, and yoga straps were used as needed for postures (though most participants opted to not use the strap). Yoga blocks were placed on the floor under the feet of participants whose feet did not reach the ground. Modifications and assists were made using verbal cues and modeling with no hands-on assists implemented though participants did receive assistance with props as needed. An externally hired C-IAYT, who was trained to deliver the intervention and not improvise, served as the interventionist at facility $A$, and the PI served as the interventionist in facilities $B, C$, and $D$. To ensure treatment fidelity, the same research assistant completed fidelity checks across all sites. The fidelity check consisted of a list of each yoga component in the order they occurred. When the research assistant was not able to attend in person, a video recording was used to complete the checklist. Any deviations from the planned program were noted and explained.

\subsection{Data Collection}

\subsubsection{Quantitative}

At baseline, participants completed demographic questionnaires that included information on gender, age, marital status, race, education level, general health information, previous yoga experience, and length of stay at the ALF. After the conclusion of the eight-week intervention, participants completed an anonymous eight-item satisfaction survey with questions that addressed different aspects of feasibility such as: "how likely are you to continue practicing yoga," "how satisfied were you with the overall class," and "how often did you feel safe during classes," Responses were reported on a 5-point Likert scale with a higher score indicating greater satisfaction. Throughout the intervention, directed field notes (e.g., predetermined quantitative information such as attendance and fidelity checklists) were completed through observational data collected by a member of the research team $[36,39]$.

\subsubsection{Qualitative}

The present analysis used reflective field notes and focus group data to answer the research question. Reflective field notes (e.g., qualitative open-ended reflections on behaviors or quotes from participants and staff) were written during the recruitment process, and immediately after each intervention session.

After the eight-week intervention, a research team member who had not attended the bi-weekly sessions facilitated an in-person focus group at each facility (Facility $A, n=4$; Facility $B, n=4$; Facility 
C, $n=3$; Facility $D, n=4)$. Participants were asked to answer eleven open-ended questions that focused on feasibility and perceived outcomes (see Table 3 for full list of feasibility-related focus group question). Additional prompts were utilized to probe for data that specifically addressed the feasibility constructs [40].

Table 3 Feasibility focus group questions.

\begin{tabular}{ll}
\hline Focus Group Question & Aspect of Feasibility \\
\hline $\begin{array}{l}\text { Describe what you were expecting when you signed up for } \\
\text { the program. }\end{array}$ & Reach \\
What were you hoping to get out of the program? & Reach \\
$\begin{array}{l}\text { Describe any concerns you had before we started, or } \\
\text { anything you thought might be difficult. }\end{array}$ & Reach \\
$\begin{array}{l}\text { Describe any elements of the yoga program you really } \\
\text { liked or disliked. }\end{array}$ & Acceptability \\
$\begin{array}{l}\text { Now that the program is completed, what are your } \\
\text { perceived benefits? }\end{array}$ & Acceptability \\
$\begin{array}{l}\text { Was there anything that you experienced that wasn't } \\
\text { positive? }\end{array}$ & Acceptability \\
$\begin{array}{l}\text { Describe any scheduling conflicts you had with the } \\
\text { program. }\end{array}$ & Practicality \\
$\begin{array}{l}\text { Describe any plans you have for continuing yoga. } \\
\begin{array}{l}\text { Describe any safety concerns you had during the } \\
\text { intervention }\end{array}\end{array}$ & Demand \\
$\begin{array}{l}\text { Is there anything else you would like us to know about } \\
\text { your experience with the program? }\end{array}$ & Occeptability \\
\hline
\end{tabular}

\subsection{Data Analysis}

All quantitative data were entered into an excel spreadsheet. IBM SPSS Statistics for Windows version 24 (IBM Corp., Armonk, N.Y., USA) was used to conduct descriptive statistics, including frequencies, means, and percentages.

Each participant was assigned a pseudonym to maintain anonymity and confidentiality. Handwritten reflective field notes were deidentified and transcribed into electronic documents. Focus groups were audio-recorded, transcribed verbatim, and de-identified. Qualitative data, 
including reflective field notes and focus groups, were stored and analyzed using NVivo 12 software. Directed content analysis is used when a conceptual theory or framework already exists [40]. This method was used in this study to analyze the qualitative data based on aspects of feasibility. Text from the focus groups were initially assigned to six predetermined feasibility codes that corresponded with the components of feasibility which are outlined in Table 1.

After the analysis was complete, an external auditor independently reviewed the data and coding to ensure consistent interpretation; there was over $90 \%$ agreement between the external auditor and $\mathrm{PI}$, and the discrepancies were re-evaluated until a consensus was reached. After initial data analysis, member checking occurred in person by the PI meeting with participants and providing a written and verbal summary of the findings. Eleven of 15 participants engaged in member checking; they were asked to verbally provide feedback, and were also provided a form where they could anonymously indicate the extent to which they agreed with the results of the study. Additionally, there was space for open-ended written feedback. All participants who responded confirmed they agreed with the results of the study, and did not provide additional verbal or written feedback. Throughout the research process, the PI engaged in bracketing and this continued throughout data analysis to help ensure trustworthiness.

Data mixing took place after the initial data analyses to compare quantitative and qualitative findings. Quantitative outcomes and qualitative themes were used to triangulate findings and determine which results were convergent and divergent for each of the predetermined themes [41].

\subsubsection{Reach}

The percent of the population reached was calculated by dividing the number of study participants by the total number of residents at each facility [39]. Percent of total residents who enrolled in the study was calculated. To meet the benchmark of feasibility, at least $13.2 \%$ of residents should have enrolled across the four facilities [19]. Next, a one proportion Z-test was used to determine if there were any significant differences in demographics between the study sample and the national census [42]. Predetermined codes used for qualitative data analysis specific to reach included initial expectations, initial concerns, and motivations to participate.

\subsubsection{Demand}

Demand represents the extent to which the target population is likely to use the intervention. Researchers calculated attrition and attendance rates from information in the directed field notes. Benchmarks for demand were set at no more than $20 \%$ attrition and at least $50 \%$ attendance for each enrolled participant. The percentage of participants who reported they were likely (4) or very likely (5) to continue practicing yoga was calculated based on the satisfaction survey responses. At least $90 \%$ of participants are expected to report being likely or very likely to continue participation in yoga to meet the feasibility benchmark. Predetermined codes for qualitative data analysis related to demand included intent to continue yoga practice and current independent yoga practice.

\subsubsection{Acceptability}

Acceptability describes how appealing and appropriate an intervention is to a population. Researchers analyzed overall satisfaction by calculating the percentage of people who were 
reported being either satisfied (4) or very satisfied (5) with the yoga intervention. To be considered feasible, the benchmark required that at least $90 \%$ of participants responded they were satisfied or very satisfied. Predetermined codes for qualitative data analysis of acceptability were positive and negative programmatic components and perceived outcomes.

\subsubsection{Practicality}

Practicality consists of any factors that add to the ease or difficulty of implementing an intervention with the resources and the environment as they exist (without altering to create a controlled environment for research). Researchers recorded the resources (i.e., staff time and equipment) used and the number of scheduling conflicts in directed field notes. Reflective field notes recorded generally anything that contributed to the ease or difficulty of implementation from the perspective of the research team, including the reasons for scheduling conflicts when participants missed sessions. Predetermined codes for qualitative data analysis specific to practicality were facilitators for and barriers to implementation.

\subsubsection{Treatment Fidelity}

A trained research assistant observed each session, assessing for fidelity. Researchers evaluated to what extent the implemented intervention adhered to the protocol by calculating the percent change between planned and observed session length, the total number of sessions delivered, and yoga postures included in the practice. To be considered feasible, there should be no more than $20 \%$ deviation from the protocol in any area. Predetermined codes for qualitative data analysis related to treatment fidelity included justification of modifications to the protocol.

\subsubsection{Treatment Fidelity}

Researchers documented the number of adverse events, or potential adverse events that occurred during the intervention. A benchmark of less than $10 \%$ of participants experiencing an adverse event or near-miss event was used to determine feasibility. Additionally, at least $90 \%$ of participants should report feeling safe almost always (4) or always (5) in response to the question "how often did you feel safe?" on the satisfaction survey. The predetermined code for safety used for qualitative data analysis was perceived safety.

\section{Results}

\subsection{Reach}

Out of the total 126 residents across all four facilities, 17 (13.49\%) were successfully enrolled in the study, and 15 successfully completed the intervention. There were 109 individuals who met the initial screening criteria (i.e., they had no known diagnosis of dementia, and no exercise restrictions), 29 individuals were willing to sign the informed consent and were screened to further determine whether they met inclusion criteria, 17 were eligible to enroll, and 15 completed post-testing (see Table 2 for the full information about recruitment and enrollment). Two participants were lost to follow up: one was discharged to the hospital, and one passed away. 
The average age of study participants who began the intervention was $82.94 \pm 9.01$ and their mean length of stay at the ALF was $1.07 \pm 2.01$ years. All participants were Caucasian, 15 were female, and none had experience practicing yoga. There was no statistically significant difference in demographics, health conditions, or length of stay between our sample and national census data (see Table 4 for additional demographic information about the sample and comparison with the national census). This met the previously determined benchmark for feasibility of reach. Residents who declined to participate in the research study were allowed to attend the yoga class; on average 3.97 non-study participants were in attendance.

Table 4 Sample demographics versus national demographics.

\begin{tabular}{|c|c|c|c|}
\hline & \multicolumn{2}{|c|}{ Sample number (\%) } & \multirow[t]{2}{*}{ National \% } \\
\hline & Pre-test $(n=17)$ & Post-test $(n=15)$ & \\
\hline \multicolumn{4}{|l|}{ Age } \\
\hline$<65$ & $2(12 \%)$ & $2(13 \%)$ & 7 \\
\hline $65-74$ & $2(12 \%)$ & $2(13 \%)$ & 11 \\
\hline $75-84$ & $4(23 \%)$ & $4(27 \%)$ & 30 \\
\hline $85<$ & $9(53 \%)$ & $7(47 \%)$ & 52 \\
\hline \multicolumn{4}{|l|}{ Race } \\
\hline Caucasian & 17 (100\%) & 15 (100\%) & 81 \\
\hline \multicolumn{4}{|l|}{ Gender } \\
\hline Female & $15(88 \%)$ & $13(87 \%)$ & 74 \\
\hline Male & $2(12 \%)$ & $2(13 \%)$ & 26 \\
\hline \multicolumn{4}{|l|}{ Education } \\
\hline High school or less & $9(53 \%)$ & $8(53 \%)$ & 59 \\
\hline Some college or higher & $8(47 \%)$ & $7(47 \%)$ & 41 \\
\hline \multicolumn{4}{|l|}{ Marital Status } \\
\hline Single (never married) & $1(6 \%)$ & $1(7 \%)$ & 14 \\
\hline Currently married & $3(18 \%)$ & $3(20 \%)$ & 13 \\
\hline Widowed & 10 (59\%) & $8(53 \%)$ & 63 \\
\hline Divorced/separated & $3(18 \%)$ & $3(20 \%)$ & 10 \\
\hline \multicolumn{4}{|l|}{ Overall health } \\
\hline Excellent & $2(12 \%)$ & $2(13 \%)$ & 5 \\
\hline Very good & $4(24 \%)$ & $4(27 \%)$ & 16 \\
\hline Good & $5(29 \%)$ & $5(33 \%)$ & 38 \\
\hline Fair & $5(29 \%)$ & $4(27 \%)$ & 32 \\
\hline Poor & $1(6 \%)$ & $0(0 \%)$ & 10 \\
\hline \multicolumn{4}{|l|}{ Health conditions } \\
\hline High blood pressure & $7(41 \%)$ & $7(47 \%)$ & 52 \\
\hline Arthritis & $5(29 \%)$ & $5(33 \%)$ & 42 \\
\hline Mental health & $5(29 \%)$ & $5(33 \%)$ & 31 \\
\hline \multicolumn{4}{|l|}{ Length of Stay } \\
\hline Less than one year & 7 (41\%) & $7(47 \%)$ & 32 \\
\hline $1-2$ years & $5(29 \%)$ & $4(27 \%)$ & 35 \\
\hline
\end{tabular}




\begin{tabular}{llll}
\hline $3-4$ years & $4(24 \%)$ & $3(20 \%)$ & 16 \\
$5+$ years & $1(6 \%)$ & $1(7 \%)$ & 15 \\
\hline
\end{tabular}

National average information was retrieved from Center for Health Statistics,(2013)

In the focus group, participants discussed their initial expectations, initial concerns, and their motivation/s for signing up for the program. Most participants were motivated by curiosity or a desire to stay involved in the activities offered at the facility. Field notes indicated that central concerns for those who declined to participate were that they would not be able to do the yoga or that it conflicted with their religious beliefs. For those who consented, the primary concern was that they did not know what to expect. When discussing initial concerns, Charles shared "Somebody had told me it was a religious experience...I don't see it that way but... some said: Beware..." and Danna discussed her apprehension saying "...of course I was thinking we might do some weird stuff..." Overall, the two data strands were convergent and indicated that the reach of the intervention was feasible.

\subsection{Demand}

The overall attrition rate was $11.76 \%$. Participants attended, on average, $12.69 \pm 2.06$ of 16 offered yoga sessions (79.31\%) with a minimum of nine and a maximum of 15 sessions out of the 16 sessions. Of those who completed the study, nine of the 15 had good attendance $(>80 \%)$, and six had moderate attendance (between $20 \%-80 \%$ ). Out of the 272 potential instances of attendance, there were 68 total absences which met the benchmark of at least $50 \%$ attendance. Fifty-five absences (20.22\%) were related to scheduling conflicts (discussed under practicality), and 13 (4.78\%) were related to feeling ill, autonomously declining to participate, or not being present at the facility for reasons unknown. When asked about plans to continue yoga, all participants indicated that they would continue participation; nine participants proactively requested that the program continue. For example, Darlene described her desire for the program to continue by saying "...I hope they have somebody else come and do this [teach yoga] ...please set it up [continued yoga group] and come and get me!" Charles, a resident in a different facility described his desire to continue his yoga practice saying "I don't want this to end. I feel better the rest of the day. I can let things not bother me. I feel like my whole body is awake."

The other six participants stated that they would attend, but were more passive in their intent to participate. For example, Aletta said, “...I ain't got nothin' else to do. I just go along with the program..." Bonnie stated "I'm just gonna be honest...I don't care for yoga much..." When prompted to expand on disliking yoga, she stated, "Well, I don't know. I guess I just kind of feel like I get enough exercise on my own, but I'll be there next time." Yet, in focus groups she indicated that she enjoyed the activity because of the social aspect of attending and would continue to participate because she thought it was important to take advantage of opportunities to move.

The two data strands indicate the intervention was feasible. The quantitative data met the benchmarks, and the qualitative data were convergent with the quantitative results, even though qualitative responses indicated varying degrees of demand from participants. 


\subsection{Acceptability}

Anonymous satisfaction surveys were completed by 12 of 15 participants. Eight reported being satisfied, three reported being very satisfied, and one reported being very unsatisfied with the overall program. The one who reported being very unsatisfied indicated they were very unsatisfied with the level of difficulty of the class, but indicated that they are very likely to try yoga again, and that they were very satisfied with all other aspects of the intervention. These scores met the benchmark for acceptability.

In the focus group, participants discussed their perceived benefits, which included improved relaxation $(n=11)$, maintaining or improving fitness $(n=8)$, opportunity for social interaction $(n=5)$, and improved breathing $(n=4)$. Some participants discussed perceived benefits. Abigail said: "It seemed like I got stronger." Others highlighted the social benefits. For example, Darlene said: "That's a great way to make new friends." In the field notes, the researcher noted that participants engaged and appeared to enjoy the mantra portion of the yoga sequence during five sessions, writing "All participants seem to consistently enjoy repeating the mantra out loud." The field notes also quoted a participant that said, "When I say the mantra, I can do more." Participants did not identify any specific aspects of the yoga practice that they disliked.

Together, the two data strands indicate that the intervention was acceptable. The participants were satisfied with the intervention and perceived positive outcomes in an ALF setting.

\subsection{Practicality}

Practicality consisted of documenting the factors that impacted the ease or difficulty of implementing the intervention. Identified factors included adequate space, disruptions, and scheduling conflicts.

\subsubsection{Interruptions}

Disruptions were noted 19 times in field notes; nine disruptions were caused by residents who were not part of the study, and 10 disruptions were caused by staff. At times, other residents walked through the group, tried to observe and/or comment on the group without participating, tried to talk to participants, or tried to use the space for a different activity. For example, the field notes reported: “Gentleman stated 'I don't understand, and I don't like it.' He was told he didn't have to stay and began whistling very loudly.... resident's whistling was very disruptive to the group. One participant said, 'One person has to spoil it for the whole group.'” In another facility, the dining room was used for yoga and field notes reported "a group of residents came in for dinner at 4:30 despite yoga therapist asking them not to and explaining the dining room wasn't open until five p.m." Field notes also report one participant responding saying, "this [residents coming in for dinner] disturbs my peace," and, "This really upsets me." In the enclosed dining room, a non-study participant who had a history of physical aggression joined the group and moved within inches of participants' faces trying to talk to people. The yoga therapist repeatedly helped her move her wheelchair out to a spot at the edge of the circle and invited her to participate, but the resident persistently tried to talk to participants during the group, creating a disruption.

In these situations, facility staff were either not present to assist in deescalating the situation or chose not to engage in managing the behavior. Other times staff caused the disruptions by 
administering medications, talking to residents, loudly encouraging the group to work harder, setting up the room for future activities or meals, or making themselves a snack. In open spaces, staff were often loudly talking as they walked by the intervention location, which many participants reported as distracting.

\subsubsection{Adequate Space}

Adequate space was an important component of the program. Aspects of adequate space included enough square footage for participants to practice, chairs already set up or nearby, comfortable temperature, limited windows that would create a glare that obstructs participant's views, and minimal interruptions by staff or other residents. Three of the four facilities had adequate space where the intervention was conducted. The setting in Facility A was in a high traffic area that had too many interruptions by both staff and other residents.

\subsubsection{Scheduling Conflicts}

Finally, excessive scheduling conflicts negatively impacted the implementation. Field notes indicated that all participants missed at least one session; there were 30 sessions missed due to scheduling conflicts, including having visitors $(n=12)$, attending physical therapy $(n=9)$, being out of the facility for a doctor's appointment $(n=6)$, concurrent facility sponsored activity $(n=2)$, and hospital admission $(n=1)$.

\subsection{Treatment Fidelity and Adaptations}

Adaptations to the protocol occurred by increasing the number of sites in order to reach our target sample size (originally one site had been chosen), and by shortening the length of individual sessions to accommodate facility scheduling and participant attention. The two instructors followed the same sequence and discussed modifications of postures, and ways to modify the sequence to fit shortened time as needed. The mean length of each session was 48:16 \pm 6:35 minutes (instead of the pre-determined 60-minute length) with a minimum of 35 minutes and a maximum of 60 minutes. Field notes reported that in one location the "Activities director asked [yoga] therapist to shorten intervention to 30 minutes from here on out due to 'attention span' of the residents." There were several occasions where another activity had been planned for the same space during the 60 minutes allotted for yoga, and at times, staff interrupted to prepare space during the yoga class. Residents also expressed a desire for shorter classes at times. Field notes report "Anna [study participant] said the yoga class is listed as being $30 \mathrm{~min}$ [sic] long in the 'program.' I'm not sure what she is referring to. I told her that it is meant to be an hour and she said, 'that is too long.'" On several occasions, participants left at 45 or 50 minutes to use the restroom even if the class continued for the full 60 minutes. However, all residents stayed for the duration when the intervention was shortened to 45-50 minutes. Thus, the session length was shortened to 50 minutes to encourage participant engagement. The total time was reduced by reducing the number of repetitions in the strength building postures from three to two repetitions.

Modifications to the standard chair yoga poses included modifying movements for a smaller range of motion and encouraging mental practice for those with limited mobility, injury, or pain. All 16 sessions were implemented in each facility, and each session contained all the components of 
the yoga sequence. In summary, the yoga sequence was delivered as designed, for 16 sessions, but the length of the sessions were reduced, and eventually, the shorter time was adopted.

\subsection{Treatment Fidelity and Adaptations}

No serious adverse events occurred in any intervention. One person complained of shoulder pain once during a session, but indicated the pain was chronic and was present before the session began. On the satisfaction survey, 11 of 12 participants indicated that they felt safe always or almost always.

During the focus groups, participants emphasized that they felt safe because they knew their own limits, and because they trusted the instructor. For example, Bonnie discussed knowing her limits saying: "...I know what I can do...so when I reach that point, I stop..." Others emphasized the importance of the instructor prioritizing safety, having adequate knowledge of specific health conditions, and offering modifications. Danna discussed the importance of the instructor knowing participant's specific health conditions, saying, “...I think it's important for the instructor should [sic] know what each person in here is capable of. Like I'm sure [Instructor's name] was well aware of what I could do, she knew what I could do ...what I couldn't do..." Charles discussed how modifications were helpful to create a feeling of safety: "...I was worried initially that I might pull a muscle or something like that. That never happened and with [Instructor's Name], she sort of said we don't want anybody getting hurt here... if you can't do it, I'll give you another way to do it. I felt secure with that."

While modifications were an important aspect of providing a safe and effective intervention, field notes indicate that suggested modifications were often ignored or not heard by participants. For example, the participant who experienced shoulder pain was encouraged to raise her arms only to shoulder height rather than overhead, yet she would often continue to lift arms overhead even when the instructor was modeling the modification. Another participant had limited use of her left side and was encouraged to engage in a mental practice, but would often complete repetitions twice on her right side. Ultimately, the principal safety concerns identified in the reflective field notes were not related to yoga postures, but the potential conflict between residents as previously discussed under practicality. However, no adverse or potential adverse events occurred, and the intervention met the benchmarks for safety.

\section{Discussion}

This convergent mixed methods research study explored the feasibility of implementing a chair yoga intervention in ALFs by evaluating the reach, demand, acceptability, practicality, treatment fidelity, and safety. Overall, quantitative data indicated that the intervention met the established benchmarks for feasibility. Results of the qualitative data (reflective field notes and focus groups) analysis were convergent with quantitative findings (see Table 5 for a summary of the findings). 
Table 5 Summary of quantitative and qualitative feasibility results.

\begin{tabular}{|c|c|c|c|c|c|c|}
\hline $\begin{array}{l}\text { Aspect of } \\
\text { Feasibility }\end{array}$ & Quantitative Measures & Benchmark & $\begin{array}{l}\text { Quantitative } \\
\text { Results }\end{array}$ & Qualitative Results & Convergent? & $\begin{array}{l}\text { Overall } \\
\text { Feasible? }\end{array}$ \\
\hline Reach & $\begin{array}{l}\% \text { residents enrolled } \\
\% \text { of those screened } \\
\text { enrolled }\end{array}$ & $\begin{array}{l}\geq 13.2 \% \\
\geq 30 \%\end{array}$ & $\begin{array}{l}* 13.49 \% \\
* 59 \%\end{array}$ & $\begin{array}{l}\text { Most were willing to try, but some held } \\
\text { beliefs about exercise or religious beliefs } \\
\text { preventing them from enrolling. }\end{array}$ & Yes & Yes \\
\hline$\underline{\text { Demand }}$ & $\begin{array}{l}\text { Attendance } \\
\text { Attrition }\end{array}$ & $\begin{array}{l}\geq 50 \% \\
\leq 20 \%\end{array}$ & $\begin{array}{l}* 75 \% \\
* 12 \%\end{array}$ & $\begin{array}{l}\text { All participants indicated they would like } \\
\text { to continue yoga; } 50 \% \text { were actively } \\
\text { trying to plan to continue to practice. } \\
\text { Other } 50 \% \text { said they would like to if } \\
\text { someone else planned it. }\end{array}$ & Yes & Yes \\
\hline Practicality & Scheduling conflicts & NA & NA & $\begin{array}{l}\text { Staff support had the biggest influence on } \\
\text { practicality in terms of interruptions and } \\
\text { scheduling conflicts. }\end{array}$ & NA & $\begin{array}{l}\text { Dependent } \\
\text { on the } \\
\text { facility }\end{array}$ \\
\hline$\frac{\text { Treatment }}{\text { Fidelity }}$ & $\begin{array}{l}\text { Actual vs. planned \# of } \\
\text { sessions }\end{array}$ & $\leq 20 \%$ & $* 0 \%$ & $\begin{array}{l}\text { Staff support had the biggest influence on } \\
\text { treatment fidelity. }\end{array}$ & Yes & Yes \\
\hline
\end{tabular}


OBM Geriatrics 2101; 5(1), doi:10.21926/obm.geriatr.2101148

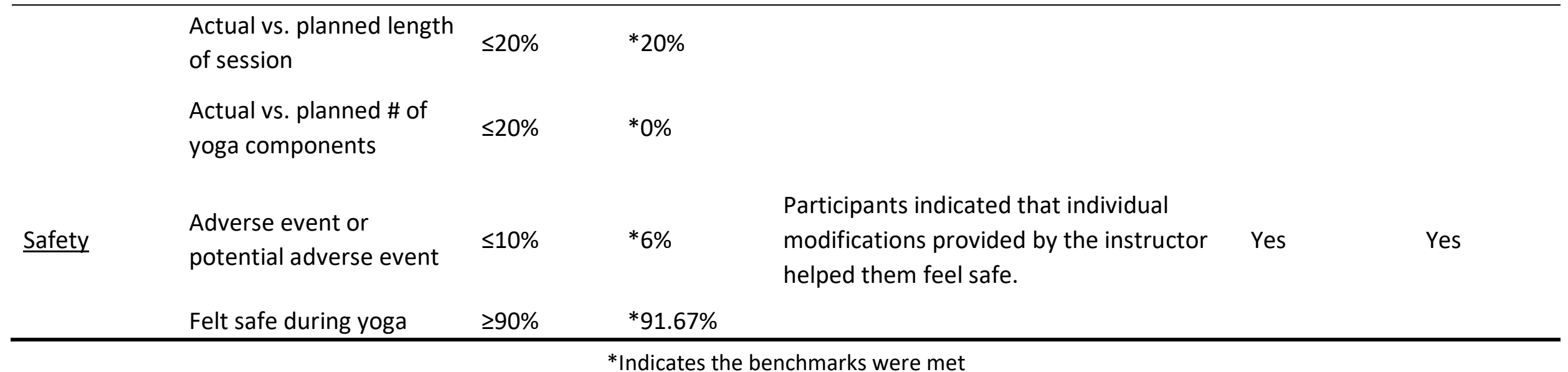


Quantitative data for reach (percentage of residents enrolled in the study) demonstrated that the intervention was feasible and qualitative data were convergent with those findings. The study sample did not significantly differ from the national census of LTC residents [43], which indicates that the reach was not specific to a subset of demographic characteristics or people with certain health conditions. However, our group was homogenous in terms of race; this was not significantly different from the national average, but acceptability may be different if working with a more diverse sample in terms of race/ethnicity. Recruitment was influenced both by general attitudes toward exercise, and preconceived notions of yoga. Most residents considered yoga a form of exercise, and therefore, those who were not interested in or confident in their ability to exercise may have self-selected out of the study. For those who enrolled, most were motivated by wanting to improve general well-being or reduce stress which is consistent with Cramer et al.'s [19], report on why people participate in yoga. Many residents who declined to participate believed yoga would be far too challenging. Freeman et al [44]. found that media depictions of yoga focus on the physical postures more than $80 \%$ of the time and tend to overlook the other components of yoga. This overemphasis may lead to a misconception that yoga is inaccessible for certain ages or ability levels. Conversely, some participants recognized that yoga involved more than postures, but considered yoga a religious practice and were more cautious about joining the study. Patel et al [45]. also found that older adults perceived yoga as a contradiction to their religion, which presented a barrier to recruitment and continued participation in a yoga study. In the present study, many participants who were outspoken about their religious views never expressed concern about any conflicting beliefs.

Quantitative data for demand (attendance, intent to continue participation) indicated that the intervention was feasible and qualitative data were convergent with those findings. Participants attended regularly, and several residents who were not part of the study also attended regularly. Overall attendance remained steady throughout the course of the intervention, which is similar to attendance rates for a yoga study at a retirement center [46], and to the attendance of a yoga study conducted with older adults with mobility limitations [47]. Attrition rates were low (11.7\%), which was comparable to findings by Hawkins et al. [46], and Groessel et al. [47] for older adults in yoga studies. Additionally, participants indicated that they wanted to continue to practice, but most wanted a group leader to run the sessions because they did not feel capable of practicing independently. This is similar to results from a yoga intervention in a continuing education center where participants indicated that they were unhappy with the intervention ending and had a desire to continue the group classes [48].

Quantitative data for acceptability (satisfaction scores) demonstrated that the intervention was feasible and qualitative data were convergent with those findings. Generally, participants responded positively to the intervention and reported positive outcomes related to social connection, stress reduction, and functional fitness. While social connection is a common outcome in yoga research [49-53], it is often attributed to meeting new people who may have the same health condition. This study demonstrates the importance of social connection, even when participants knew each other and already engaged in other activities together on a regular basis. This is congruent with research on the potential for deepening feelings of social connection using synchronous movement [54], and it might be that participants felt a deeper connection with peers when moving synchronously. However, further research is needed to explore the potential link between synchronous movement and social connection among older adults. 
Practicality results were highly dependent on specific aspects of ALFs with staff support having a substantial influence on the availability of adequate space, number of disruptions, and potential for scheduling conflicts. While yoga is practiced in a wide range of settings and spaces, the findings from the present study indicate that having adequate space reduced the risk of disruptions either by a physical enclosure or by staff who redirected potential disruptions. Staff support was central to increasing attendance, engagement, and reducing interruptions from staff and residents, and encouraging participation.

Treatment fidelity met the benchmarks for feasibility and qualitative data were convergent. It is worth reemphasizing that the protocol itself was adaptive yoga with the intention of modifying for all ability levels, which allowed the intervention to be implemented with the intended components. Sessions were initially planned for 60-minutes, but results indicated that 45-50 minutes was a more appropriate duration for participants to comfortably complete the intervention without losing attention or having people leave early. This level of tolerance may be related to cognitive impairments. In Chen et al's. [32] implementation of yoga for people with dementia, they shortened their standard 70-minute intervention to 55-minutes due to cognitive impairment. In the present study the duration was shortened at the request of ALF staff and participants.

Quantitative data indicated the intervention was safe and results from the qualitative data were convergent. Participants indicated that they felt safe throughout the yoga intervention and attributed their perceived safety to knowing the interventionist was aware of their specific health concerns and was skilled in modifying the intervention accordingly. While yoga is often seen as beneficial, it is can also be the source of discomfort, and adults over 65 are at a greater risk of yogarelated injury than the general population [55]. When working with vulnerable populations, it is imperative that the instructor has the medical knowledge to ensure safety, perceived safety, and knowledge of adapting poses so residents can receive the full benefit of the intervention [44]. Multiple studies have concurred that having a trained interventionist is vital for consistent successful outcomes and prevention of injury [35, 55-57]. The results of the present study echo the importance of having a qualified instructor lead the intervention.

Participants placed substantial importance on the instructor's ability to prevent injury. Therefore, it is our recommendation that instructors complete a 200-hour yoga training and engage in a personal yoga practice before implementing a yoga intervention. This level of training demonstrates minimum competency regarding knowledge of yoga techniques, anatomy, physiology, and biomechanics, and contraindications for specific poses [58]. Additionally, it is recommended that instructors are familiar with common health conditions among older adults, and have the skill to modify yoga postures to prevent injury and promote the most benefit.

The potential for resident conflict was noted under practicality; however, the potential for escalating conflict was also a potential safety concern [56]. While two of the four facilities had staff who supported and observed each session, two of the facilities left the interventionist alone with participants. There were multiple instances of verbal conflict between participants at these facilities. This highlights the importance of adequately training staff to understand the nature of yoga, encourage participation, and de-escalating verbal conflicts during the intervention. Additionally, this study revealed a trained interventionist cannot compensate for lack of staff support and involvement. Facility staff played a vital role in gathering residents and deescalating agitated residents. Results from the present study indicate that a yoga intervention is feasible in ALFs, but 
future studies could examine specific outcomes, and determine if it is more beneficial than other activities.

\section{Conclusion}

\subsection{Implications and Future Research}

Overall, this study found that offering yoga in an ALF is feasible. However, ALFs should consider the training of the interventionist, adequate space, and direct care staff support when planning a yoga intervention. Prior to implementing a yoga intervention, we recommend educating all staff on the practice, benefits, and etiquette of yoga. Future research might explore comparing a verbal training with an experiential training where staff participate in yoga to see if their support changes after experiencing the intervention. Additionally, future research might explore using signage to request privacy and silence during a yoga group as a reminder to both staff and other residents.

\subsection{Limitations}

This was a small, convenience sample of ALFs. The feasibility was reflective not only of the acceptability of yoga, but also of the acceptability of conducting research within ALFs. The fact that several residents attended the sessions who were not willing to participate in research, indicates that feasibility results may be improved if the research component was removed. It is possible that social desirability influenced some answers provided by participants, and this was mitigated by gathering data from a variety of sources.

\subsection{Summary}

To our knowledge, this is the first article that explores the feasibility of implementing a yoga intervention in ALFs. Findings indicate that it is feasible in terms of reach, acceptability, demand, treatment fidelity, and safety. Finally, practicality results indicate the intervention is practical overall, but may be highly influenced by staff support at individual facilities.

\section{Author Contributions}

Em V. Adams designed the study, conducted the data collection, assisted in implementing the intervention, and was the primary author. Marieke Van Puymbroeck assisted in developing the study, provided council during recruitment, and assisted in writing and editing many drafts of the manuscript. Brandi M. Crowe assisted in designing the study, provided guidance through data analysis, and edited several drafts of the manuscript. Claire K. Allison assessed in data collection, implementing the intervention, and in editing, the manuscript. Cynthia L.M. Pury provided feedback for both the study design and the manuscript. Arlene A. Schmid provided feedback on the study design and edited several drafts of the manuscript.

\section{Competing Interests}

The authors have declared that no competing interests exist. 


\section{References}

1. Caregiving and assisted living facility information [Internet]. Washington: American Association for Retired Persons; 2019 [2019 October 22] Available from: https://www.aarp.org/caregiving/basics/info-2017/assisted-living-options.html.

2. Ortman JM, Velkoff VA, Hogan H. An aging nation: The older population in the United States. Final report. Washington: United States Census Bureau, Economics and Statistics Administration, US Department of Commerce; 2014.

3. Gawande A. Being mortal: Medicine and what matters in the end. New York: Metropolitan Books; 2014.

4. Fox $P$, Raina $P$, Jadad $A R$. Prevalence and treatment of pain in older adults in nursing homes and other long-term care institutions: A systematic review. CMAJ. 1999; 160: 329-333.

5. Lukas A, Mayer B, Fialová D, Topinkova E, Gindin J, Onder G, et al. Pain characteristics and pain control in European nursing homes: Cross-sectional and longitudinal results from the Services and Health for Elderly in Long TERm care (SHELTER) study. J Am Med Dir Assoc. 2013; 14: 421428.

6. Milanović Z, Pantelić S, Trajković N, Sporiš G, Kostić R, James N. Age-related decrease in physical activity and functional fitness among elderly men and women. Clin Interv Aging. 2013; 8: 549556.

7. Król-Zielińska M, Kusy K, Zieliński J, Osiński W. Physical activity and functional fitness in institutionalized vs. independently living elderly: A comparison of 70-80-year-old city-dwellers. Arch Gerontol Geriatr. 2011; 53: e10-e16.

8. Howie LO, Troutman-Jordan M, Newman AM. Social support and successful aging in assisted living residents. Educ Gerontol. 2014; 40: 61-70.

9. 2018 Program Requirements Medicare [Internet]. Baltimore: Centers for Medicare \& Medicaid Services; 2020 [2020 January 17]. Available from: https://www.cms.gov/Regulations-andGuidance/Legislation/EHRIncentivePrograms/2018ProgramRequirementsMedicare.

10. Iyengar BK. Light on yoga. 9th ed. India: Harper Collins; 2000.

11. Büssing A, Ostermann $T$, Ludtke R, Michalsen A. Impact of yoga on chronic pain-and painassociated disability: A meta-analysis. J Pain. 2012; 13: 1-9.

12. Boslego LA, Phillips CE, Atler KE, Tracy BL, Van Puymbroeck M, Schmid AA. Impact of yoga on balance, balance confidence and occupational performance for adults with diabetic peripheral neuropathy: A pilot study. Br J Occup Ther. 2017; 80: 155-162.

13. Schmid AA, Van Puymbroeck M, Portz JD, Atler KE, Fruhauf CA. Merging yoga and occupational therapy (MY-OT): A feasibility and pilot study. Complement Ther Med. 2016; 28: 44-49.

14. Van Puymbroeck M, Atler K, Portz JD, Schmid AA. Multidimensional improvements in health following Hatha Yoga for individuals with diabetic peripheral neuropathy. Int J Yoga Therap. 2018; 28: 71-78.

15. Youkhana S, Dean CM, Wolff M, Sherrington C, Tiedemann A. Yoga-based exercise improves balance and mobility in people aged 60 and over: A systematic review and meta-analysis. Age Ageing. 2016; 45: 21-29.

16. Patel NK, Newstead AH, Ferrer RL. The effects of yoga on physical functioning and health related quality of life in older adults: A systematic review and meta-analysis. J Altern Complement Med. 2012; 18: 902-917. 
17. Schmid AA, Van Puymbroeck M, Koceja DM. Effect of a 12-week yoga intervention on fear of falling and balance in older adults: A pilot study. Arch Phys Med Rehabil. 2010; 91: 576-583.

18. Field T, Diego M, Delgado J, Medina L. Yoga and social support reduce prenatal depression, anxiety and cortisol. J Bodyw Mov Ther. 2013; 17: 397-403.

19. Cramer H, Ward L, Steel A, Lauche R, Dobos G, Zhang Y. Prevalence, patterns, and predictors of yoga use. Am J Prev Med. 2016; 50: 230-235.

20. Portz JD, Waddington E, Atler KE, Van Puymbroeck M, Schmid AA. Self-management and yoga for older adults with chronic stroke: A mixed-methods study of physical fitness and physical activity. Clin Gerontol. 2018; 41: 374-381.

21. Schmid AA, Miller KK, Van Puymbroeck M, Debaun-sprague E. Yoga leads to multiple physical improvements after stroke, a pilot study. Complement Ther Med. 2014; 22: 994-1000.

22. Schmid AA, Van Puymbroeck M, Altenburger PA, Dierks TA, Miller KK, Damush TM, et al. Balance and balance self-efficacy are associated with activity and participation after stroke: $A$ cross-sectional study in people with chronic stroke. Arch Phys Med Rehabil. 2012; 93: 11011107.

23. Colgrove YS, Sharma N, Kludlng P, Potter D, Imming K, VandeHoef J. Effect of yoga on motor function in people with Parkinson's Disease: A randomized, controlled pilot study. J Yoga Phys Ther. 2012; 2: 112.

24. Van Puymbroeck, M Walter AA, Hawkins BL, Sharp JL, Woschkolup K, Urrea-Mendoza E, et al. Functional improvements in Parkinson's disease following a randomized trial of yoga. Evid Based Complement Alternat Med. 2018; 2018: 8516351.

25. Walter AA, Adams E V., Van Puymbroeck M, Crowe BM, Urrea-Mendoza E, Hawkins BL, et al. Changes in nonmotor symptoms following an 8-week yoga intervention for people with Parkinson's disease. Int J Yoga Therap. 2019; 29: 91-99.

26. Ahmadi A, Nikbakh M, Arastoo A, Habibi AH. The effects of a yoga intervention on balance, speed and endurance of walking, fatigue and quality of life in people with multiple sclerosis. J Hum Kinet. 2010; 23: 71-78.

27. McCrane K, Pei-Chun $\mathrm{H}$. Effect of yoga on $\mathrm{QOL}$ in individuals with multiple sclerosis. Ther Recreat J. 2017; 51: 81-85.

28. Hubbard C. The influence of yoga on people with traumatic brain injury related to sleep and mood. Clemson: Clemson University. 2018.

29. Schmid AA, Miller KK, Van Puymbroeck M, Schalk N. Feasibility and results of a case study of yoga to improve physical functioning in people with chronic traumatic brain injury. Disabil Rehabil. 2016; 38: 914-920.

30. Wang D. The use of yoga for physical and mental health among older adults: A review of the literature. Int J Yoga Therap. 2009; 19: 91-96.

31. Chen KM, Chen MH, Lin MH, Fan JT, Lin HS, Li CH. Effects of yoga on sleep quality and depression in elders in assisted living facilities. J Nurs Res. 2010; 18: 53-61.

32. Chen KM, Wang $\mathrm{HH}$, $\mathrm{Li} \mathrm{CH}$, Chen MH. Community vs. institutional elders' evaluations of and preferences for yoga exercises. J Clin Nurs. 2011; 20: 1000-1007.

33. Yoga Alliance Standards [Internet]. Arlington: Yoga Alliance; 2020 [2020 December 16]. https://www.yogaalliance.org/New RYS Standards/Common_Core Curriculum for RYS 200. 
34. Chen KM, Chen MH, Chao HC, Hung HM, Lin HS, Li CH. Sleep quality, depression state, and health status of older adults after silver yoga exercises: Cluster randomized trial. Int J Nurs Stud. 2009; 46: 154-163.

35. Libby D., Reddy F, Pilver C, Dsai R. The use of yoga in specialized VA PTSD treatment programs. Int J Yoga Therap. 2012; 22: 79-88.

36. Bowen DJ, Kreuter M, Spring B, Cofta-Woerpel L, Linnan L, Weiner D, et al. How we design feasibility studies. Am J Prev Med. 2009; 36: 452-457.

37. Thomas S, Reading J, Shephard RJ. Revision of the physical activity readiness questionnaire (PAR-Q). Can J Sport Sci. 1992; 17: 338-345.

38. Borson S, Scanlan JM, Chen P, Ganguli M. The Mini-Cog as a screen for dementia: Validation in a population-based sample. J Am Geriatr Soc. 2003; 51: 1451-1454.

39. Glasgow RE, McKay HG, Piette JD, Reynolds KD. The RE-AIM framework for evaluating interventions: What can it tell us about approaches to chronic illness management? Patient Educ Couns. 2001; 44: 119-127.

40. Hsieh HF, Shannon SE. Three approaches to qualitative content analysis. Qual Health Res. 2005; 15: $1277-1288$.

41. Creswell JW, Clark VL. Designing and Conducting Mixed Methods Research. 3rd ed. Thousand Oaks: Sage Publications Inc; 2017.

42. Long-term care providers and services users in the United States: Data from the national study of long-term care providers, 2013-2014 [Internet]. Center for Health Statistics N; 2016. Available from: http://lccn.loc.gov/2016000580.

43. Long-term care providers and services users in the United States: Data from the national study of long-term care providers, 2013-2014 [Internet]. Center for Health Statistics N; 2013. Available from: http://lccn.loc.gov/2016000580.

44. Freeman $\mathrm{H}$, Vladagina $\mathrm{N}$, Razmjou $\mathrm{E}$, Brems $\mathrm{C}$. Yoga in print media: Missing the heart of the practice. Int J Yoga. 2017; 10: 160-166.

45. Patel NK, Akkihebbalu S, Espinoza SE, Chiodo LK. Perceptions of a community-based yoga intervention for older adults. Act Adapt Aging. 2011; 35: 151-163.

46. Van Puymbroeck M, Schmid A, Walter A, Hawkins B. Improving leisure constraints in older adults with a fear of falling through Hatha yoga: An acceptability and feasibility study. Int J Gerontol Geriatr Res. 2017; 1: 7-13.

47. Groessl EJ, Maiya M, Elwy AR, Riley KE, Sarkin AJ, Eisen SV, et al. The essential properties of yoga questionnaire: Development and methods. Int J Yoga Therap. 2015; 25: 51-59.

48. Adams EV., Crowe BM, Van Puymbroeck M, Alison CK, Schmid AA. Yoga as a community-based recreational therapy intervention for older adults: A pilot study. Ther Recreat J. 2019; 53.

49. Crowe BM, Van Puymbroeck M, Linder SM, Mcguire FA, \& Watt PJ. The effects of yoga participation on women's quality of life and symptom management during the menopausal transition: A pilot study. Health Care Women Int. 2015; 36: 1124-1142.

50. Lin KY, Hu YT, Chang KJ, Lin HF, Tsauo JY. Effects of yoga on psychological health, quality of life, and physical health of patients with cancer: A meta-analysis. Evid Based Complement Alternat Med. 2011; 2011: 659876.

51. Van Puymbroeck M, Burk BN, Shinew KJ, Kuhlenschmidt MC, Schmid AA. Perceived health benefits from yoga among breast cancer survivors. Am J Heal Promot. 2013; 27: 308-315. 
52. Van Puymbroeck M, Payne LL, Hsieh PC. A phase I feasibility study of yoga on the physical health and coping of informal caregivers. Evid Based Complement Alternat Med. 2007; 4: 519-529.

53. Van Puymbroeck $M$, Smith $R$, Schmid $A$. Yoga as a means to negotiate physical activity constraints in middle-aged and older adults. Int J Disabil Hum Dev. 2011; 10: 117-121.

54. Tunçgenç B, Cohen E. Movement synchrony forges social bonds across group divides. Front Psychol. 2016; 7: 782.

55. Swain TA, McGwin G. Yoga-related injuries in the United States From 2001 to 2014. Orthop J Sport Med. 2016; 4: 232596711667170.

56. Hart J. An overview of clinical applications of therapeutic yoga. Altern Complement Ther. 2008; 14: 29-32.

57. Wiles AK. An investigation into the use of yoga in Recreational Therapy practice. TigerPrints 2019.

58. RYS 200 Standards. Yoga alliance [Internet]. Arlington: Yoga Alliance; 2020. Available from: https://www.yogaalliance.org/credentialing/standards/200-hourstandards.

59. Long-term care services in the United States: 2013 overview [Internet]. Washington:National Center for Health Statistics; 2013. Available from: https://www.cdc.gov/nchs/data/nsltcp/long term care services 2013.pdf.

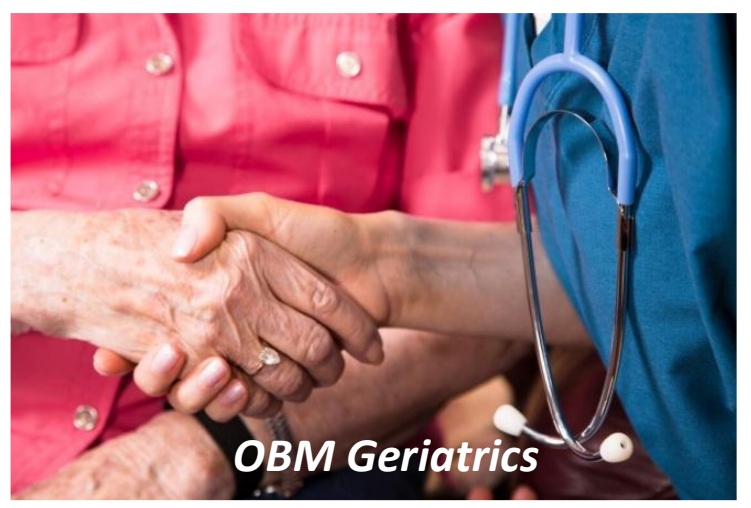

Enjoy OBM Geriatrics by:

1. Submitting a manuscript

2. Joining in volunteer reviewer bank

3. Joining Editorial Board

4. Guest editing a special issue

For more details, please visit:

http://www.lidsen.com/journals/geriatrics 